\title{
Independent Steroid Control of the Fates of Motoneurons and Their Muscles during Insect Metamorphosis ${ }^{1}$
}

\author{
JANIS C. WEEKS ${ }^{2}$ AND JAMES W. TRUMAN \\ Department of Zoology, University of Washington, Seattle, Washington 98195
}

\begin{abstract}
The metamorphosis of insects is controlled by the blood titers of a small number of developmental hormones including a class of steroids, the ecdysteroids. We have studied the developmental fates of several muscles and their motoneurons during the larval-pupal transformation of the tobacco hornworm, Manduca sexta. The endocrine events which trigger pupal development are first, a fall in the blood titer of juvenile hormone, followed by two subsequent elevations of blood ecdysteroids. The small "commitment pulse" of ecdysteroids commits tissues to pupal development, whereas the sustained "prepupal peak" causes the new pupa to be formed (Riddiford, L. M. (1980) In Progress in Ecdysone Research, J. A. Hoffmann, ed., pp. 409-430, Elsevier/NorthHolland Biomedical Press, Amsterdam). In the present experiments we were able to correlate specific aspects of the changing blood steroid titers with the degeneration of larval muscles, and with the dendritic regression and death of their motoneurons.

The abdominal prolegs, which are the principal locomotory appendages of the caterpillar, are lost during the larval-pupal transformation. We have followed the fates of a proleg retractor muscle, PPRM, and its single motoneuron, PPR. Two other differently fated abdominal muscies not associated with the proleg were also studied. Surgical and endocrinological manipulations showed that PPRM degenerates in response to the rising phase of the prepupal ecdysteroid peak and that interactions with its motoneuron are not involved in the muscle's death. Motoneuron PPR responds to the rising prepupal peak by first reducing its dendritic arbor by $40 \%$ and then dying. Other proleg motoneurons regress but do not die, indicating that dendritic regression is programmed separately from neuronal death. Neither the dendritic reduction nor the death of PPR involves interactions with its target muscle. These results indicate that ecdysteroids have independent and parallel effects in the periphery, where they cause muscle degeneration, and in the central nervous sys-
\end{abstract}

Received November 19, 1984; Revised January 22, 1985

Accepted January 24, 1985

${ }^{1}$ This research was supported by National Institutes of Health Postdoctoral Fellowship NS06605 to J. C. W., and National Institutes of Health Grant NS13079, National Science Foundation Grant PCM-8020975, and McKnight Foundation grants to J. W. T. We thank Dr. S. E. Reiss for invaluable histological assistance, and P. F. Copenhaver and Drs. R. Booker, D. B. Morton, M. A. Murray, L. M. Riddiford, and W. M. Roberts for helpful discussions.

${ }^{2}$ To whom correspondence should be sent, at her present address: Department of Entomological Sciences, Wellnan Hall, University of Califomia, Berkeley, Berkeley, CA 94720 tem, where they cause dendritic regression and death of motoneurons.

Many factors during development determine the ultimate survival, structure, and synaptic connectivity of neurons. Neuronal fates are influenced by local or contact-mediated interactions with other neurons or target tissues (Hamburger and Oppenheim, 1982; LeviMontalcini, 1982), as well as by blood-borne chemical substances which originate outside the nervous system. For instance, differences in the number and dendritic branching of neurons in several sexually dimorphic regions in the vertebrate central nervous system (CNS) depend on the blood steroids present during development (Arnold and Gorski, 1984). Steroids also infiuence the differentiation of peripheral structures such as muscles (Cihak et al., 1970), which could indirectly affect the fates of neurons.

Steroids have similar developmental effects in invertebrates, which have the advantage that endocrine effects can be studied in individually identified neurons rather than in populations of neurons. The metamorphosis of the tobacco hornworm, Manduca sexta, from caterpillar to pupa to moth is controlled by a small ensemble of developmental hormones, including a class of steroid hormones, the ecdysteroids. Although much is known about the actions of ecdysleroids on some lissues such as epidermis (Riddiford, 1981, 1985), their role in directing the fates of neurons and muscles is only beginning to be examined (Schwartz and Truman, 1984; Truman and Schwartz, 1984). Manduca is particularly well suited for such studies because the blood titers of the ecdysteroids during metamorphosis are known in more detail than for any other insect (Bollenbacher et al., 1981), developmental events occur over relatively short time periods, hormonal and surgical manipulations are easily performed, and the nervous system is amenable to intracellular recording and staining techniques (Levine and Truman, 1982; Weeks and Truman, 1984a, b).

During the larval-pupal transformation of Manduca, the locomotory appendages of the caterpillar (the abdominal prolegs) are lost. We previously described two larval retractor muscles of the proleg, PPRM (principal planta retractor muscle) and APRM (accessory planta rctractor musclc), and their respective motoneurons, PPR and APR. During the larval-pupal transformation both muscles degenerate while both motoneurons undergo substantial dendritic regression (Weeks and Truman, 1984b). Following pupation PPR dies but APR survives to adulthood. In the present experiments we examined the involvement of blood steroids in directing the fates of PPR and PPRM, and of two additional larval muscles the fates of which differ from that of PPRM. We found that, although PPR's dendritic reduction and death coincided with PPRM's degeneration, this timing was not due to interactions between them but rather to parallel and independent responses to the same blood-borne steroid signals.

\section{Materials and Methods}

Experimental animals. Larvae and pupae of the tobacco hornworm Manduca sexta, were reared individually on an artificial diet (Bell and Joachim, 
1978 ) at $26^{\circ} \mathrm{C}$ on a long day photoperiod ( $17 \mathrm{hr}$ light $/ 7 \mathrm{hr}$ dark). Lights off occurred at midnight. After wandering (see Fig. 1), animals were placed into holes bored into wooden blocks to pupate. Both males and females were used for experiments. Animals were staged relative to the ecdysis to the fifth lanal instar (day LO), wandering (day W), or pupal ecdysis (day PO) (see Fig. 1). Body segments were designated by number, e.g., $A_{3}$ indicates the third abdominal segment.

Electrophysiological techniques. Animals were anesthetized in $\mathrm{CO}_{2}$ gas for 10 to $20 \mathrm{~min}$, opened along the dorsal midline, and pinned upright on a wax block. The gut and fat body were removed to expose the ventral nerve cord, and the body cavity was bathed with Manduca physiological saline (Weeks and Truman, 1984a). Individual abdominal ganglia were stabilized on a platform for intracellular recording and staining (see below). Glasstipped suction electrodes were used to record from and stimulate segmental nerves. The amplification, storage, and display of recordings were conventional (see Weeks and Truman, 1984a).

The electrical responses of muscle fibers were recorded by sucking a loop of the fiber into a large-bore suction electrode, as for an en passant nerve recording. The recordings were made differentially with the inside of the pipette as the positive lead.

Histological techniques. Details of the staining techniques appear in Weeks and Truman (1984a, b). Briefly, individual motoneurons were stained by intracellular iontophoresis of $2 \% \mathrm{CoCl}_{2}$ or $100 \mathrm{~mm}$ hexaminecobaltic chitoride (Brogan an Id Pillnan, 1981) using glass micropipelle electrodes which had resistances of 20 to 40 megohms when filled with potassium acetate. Motoneuron somata were visualized by epi-illumination and pene traled under visual guidance. Motoneurons were also stained by backfilling ganglionic nerve branches with $2 \% \mathrm{CoCl}_{2}$ for 1 day. Both types of material were precipitated, silver-intensified (Bacon and Altman, 1977), dehydrated, cleared, and embedded in Canada balsam in whole mount.

Nerve branching patterns were examined in methylene blue-stained preparations. Saturated methylene blue was diluted $1: 9$ with saline and reduced with ascorbate $(0.4 \mathrm{gm} / 100 \mathrm{ml}$ stain) while stirring for $30 \mathrm{~min}$. Animals were opened dorsally, pinned flat, stained overnight, and dissected under normal saline.

Ligation and infusion of animals. The only known sources of ecdysteroids in larval Manduca are the prothoracic glands located in the first thoracic segment. To produce abdomens that were free of this endogenous source of ecdysteroids, anesthetized lanvae were ligated around the first abdominal segment with silk thread and the anterior body was cut off. Day L2 larvae weighing more than $7.5 \mathrm{gm}$ were ligated between 3:00 P.M. and 5:00 P.M. The attainment of this weight indicated that their titers of juvenile hormone had dropped and that the commitment pulse of ecdysteroids would occur that night (Nijhout and Williams, 1974; L. M. Riddiford, personal communication). Day $\mathrm{W}$ larvae (which wandered during the 3rd day after ecdysis to the fifth instar; Dominick and Truman, 1984) were ligated between 9:00 A.M. and 11:00 A.M.

Ecdysteroids were administered to some groups of isolated abdomens by infusing them with 20-hydroxyecdysone (20-HE; Sigma Chemical Co., St Louis, MO, or Rohto Pharmaceutical Co. Ltd., Osaka, Japan). 20-HE was dissolved in saline (Ephrussi and Beadle, 1936) and adjusted to a concentration of $1 \mathrm{mg} / \mathrm{ml}$ using spectrophotometric analysis (Meltzer, 1971). Isolated abdomens were anesthetized and the tip of the dorsal abdominal horn was cut off. Polyethylene tubing (PE-10; Intramedic, Parsippany, NJ) was inserted through the horn into the hemocele, tied in place with thread, and anchored with melted wax (CSC Softseal Tackiwax, Central Scientific Co., Chicago, IL). 20-HE was infused at a constant rate through I lamilton syringes driven by a variable speed syringe pump (Sage Instruments, model 352). All abdomens were infused for $12 \mathrm{hr}$ (beginning between 9:00 A.M. and 1:00 P.M.) at a rate of $5.6 \mu \mathrm{l} / \mathrm{hr}$. This paradigm has previously been shown to promote pupal development (Nijhout, 1976). Since isolated abdomens weighed between 6 and $8 \mathrm{gm}$, of which $40 \%$ is the hemolymph volume (8eckage and Riddiford, 1982), the infusion rate was between 1.8 and 2.3 $\mu \mathrm{g}$ of $20-\mathrm{HE} / \mathrm{ml}$ of hemolymph/hr. At the end of the infusion, the tubing adjacent to the dorsal horn was fused shut with heated forceps and left in place. About half of the abdomens everted their hindguts within a day of the infusion, which often led to exsanguination if the hindgut ruptured. This was prevented by cauterizing everted hindguts closed adjacent to the body.

Quantification of muscle degeneration. Muscle degeneration in Lepidoptera involves a progressive loss of muscle mass and thinning of muscle fibers (Lockshin and Willams, 1965; Schwartz and Truman, 1984). Fiber diameter measurements were made in muscles PPRM, VEO (ventral external oblique), and VIM (ventral internal medial), in acutely denervated abdomens pinned flat under saline, using an ocular micrometer in a Wild stereomicroscope at $\times 50$ magnification. PPRM and VEO were measured in segments
$A_{3}$ through $A_{6}$. Before pupation, VIM was measured only in $A_{3}$ to $A_{5}$ because in $A_{6}$ the muscle is smaller. In Manduca, as in many Lepidoptera, the VIMs in $A_{3}$ degenerate atter pupation (Finlayson, 1956); therefore, beginning on day $\mathrm{P} 1$, only fibers in $\mathrm{A}_{4}$ and $A_{5}$ were measured.

Quantification of dendritic reduction. The structure of PPR's dendritic arbor was analyzed in ganglia $A_{3}$ through $A_{6}$ after backfilling the lateral branch of the ventral nerve ( $\mathrm{VN}_{\mathrm{i}}$; nerve branches are described in Weeks and Truman $1984 a$, b) with $\mathrm{CoCl}_{2}$. The seven motoneurons stained via $\mathrm{VN}_{1}$ arborize throughout the hemineuropil ipsilateral to the filled nerve and demarcate its boundaries, whereas the opposite hemineuropil contains only the contralateral arbor of PPR (Weeks and Truman, 1984b). A drawing tube on a Leitz compound microscope (magnification $\times 530$ ) was used to draw the outline of the ipsilateral hemineuropil and to trace PPR's contralateral arbor in each ganglion.

Each drawing was covered with an acetate transparency marked with a grid of 2. $\mathrm{mm}$ squares, each corresponding to a $3.8-\mu \mathrm{m}^{2}$ area of the original material. All boxes contained within, or transected by, the outline of the stained hemineuropil were counted to give the hemineuropil area. The hemineuropil tracing was then mirror-image reversed and superimposed over the contralateral portion of the ganglion to delineate the outline of the hemineuropil containing PPR's arbor. The dendritic area of PPR was measured by counting the number of boxes containing neuronal processes which were located within the borders of the hemineuropil. A box was counted just once regardless of how many processes transccted it. This method was thus most accurate for sparse arbors and it systematically underestimated the dendritic complexity of densely branching neurons.

For each back fill, the dendritic ared was divided by hemineuropil area to give a dendritic density score, which reflected the proportion of hemineuropil covered by PPR's processes. The normalization of dendritic density with respect to hemineuropil area compensated for size differences between different ganglia due to shrinkage during histological processing. Unless otherwise noted, five neurons were analyzed for each data point. When experimental manipulations involved the entre abdomen (e.g., infusions), more than one ganglion from the abdomen was usually backfilled. However, the five neurons analyzed for each data point were always taken from five different animals.

More than 400 ganglia were backfilled, of which 70 were analyzed quantitatively. Only ganglia in which the neurons were darkly stained and showed good resolution of dendritic structure were used. Ganglia were rejected if staining appeared incomplete or if PPR's dendrites were discontinuous or distorted. Even in poorer quality material the same morphological trends seen in the analyzed fills were obvious.

Surgical removal of PPRM (PPRMx). Larvae were anesthetized by immersion in $\mathrm{H}_{2} \mathrm{O}$ for at least $30 \mathrm{~min}$ and immobilized ventral side up in a salinefilled dish. An incision was made around PPRM's insertion point in the tip of the proleg, the muscle was pulled out through the incision, and forceps were used to tear off the muscle near its dorsal end. The operated proleg was then tied off at its base with thread. Successful muscle removal was subsequently verified by dissection.

Surgical denenation of PPRM by severance of the VN. Larvae were prepared as for muscle removal. A mid-ventral incision was made over an abdominal ganglion and one $\mathrm{VN}$ was severed. The incision was closed with two or three sutures. Severance of a VN rendered the ipsilateral proleg permanently paralyzed and unresponsive to sensory stimuli.

Statistical analysis. The significance of differences between the mean values obtained in different experimental groups was tested using one-tailed or two-tailed $t$ tests. Significance was assumed when $p<0.05$.

\section{Results}

\section{Time course of developmental events during the larval-pupal transformation of Manduca}

Figure 1 summarizes the endocrine events underlying the larvalpupal transformation in Manduca. When the fifth instar larva attains a critical body mass, the blood titer of juvenile hormone falls, permitting metamorphosis to proceed (Nijhout and Williams, 1974). On day $L 3$ a small pulse of ecdysteroids appears. This "commitment pulse," which is the first appearance of ecdysteroids in the absence of juvenile hormone, commits the body tissues to pupal differentiation (Riddiford, 1980, 1985). The commitment pulse is followed on the next night by wandering behavior, when the caterpillar leaves the food plant and burrows underground to construct a pupation chamber. On day $W 1$ blood ecdysteroids again rise during the "prepupal peak," which causes the epidermis to detach from the 
Figure 1. Endocrine events during the larval-pupal transformation of Manduca. Time is in days, with the photoperiod indicated. Ecdysteroid (solid line) and juvenile hormone (dashed line) titers are taken from the data of Bollenbacher et al. (1981), B. J. Bergot (unpublished data cited in Sedlak et al., 1983, Truman et al. (1983), and L. M. Riddiford (unpublished data). Peak blood ecdysteroid values during the commitment pulse and prepupal peak are (in 20-HE equivalents) $0.07 \mu \mathrm{g} / \mathrm{ml}$ and $1.5 \mu \mathrm{g} / \mathrm{ml}$, respectively (Bollenbacher et al., 1981). The drawing at bottom left illustrates the larval prolegs, which are present as pairs on the third through sixth abdominal segments. The terminal segment bears a modified pair of prolegs which were not studied. The inset (middle drawing) indicates on a single proleg-bearing segment the location and insertion points of the primary planta retractor muscle, PPRM (see Fig. 2 for internal view). Each of the two divisions of PPRM consists of one muscle fiber (rarely, two fibers are present). Lower right: drawing of newly ecdysed pupa $L$, larva; $W$, wandering; $P$, pupa.

Figure 2. Location of larval muscles examined in this study. Each drawing shows an internal view of a proleg-bearing abdominal hemisegment $(A$, anterior; $D$, dorsal midline; $P$, posterior; $V$, ventral midline). The ventral nerve cord is shown in black in the left drawing, and the spiracle appears near the middle of each hemisegment. From left to right, the dissection reveals successively deeper (more external) muscle layers. Muscles VIM, VEO, and PPRM are shaded.

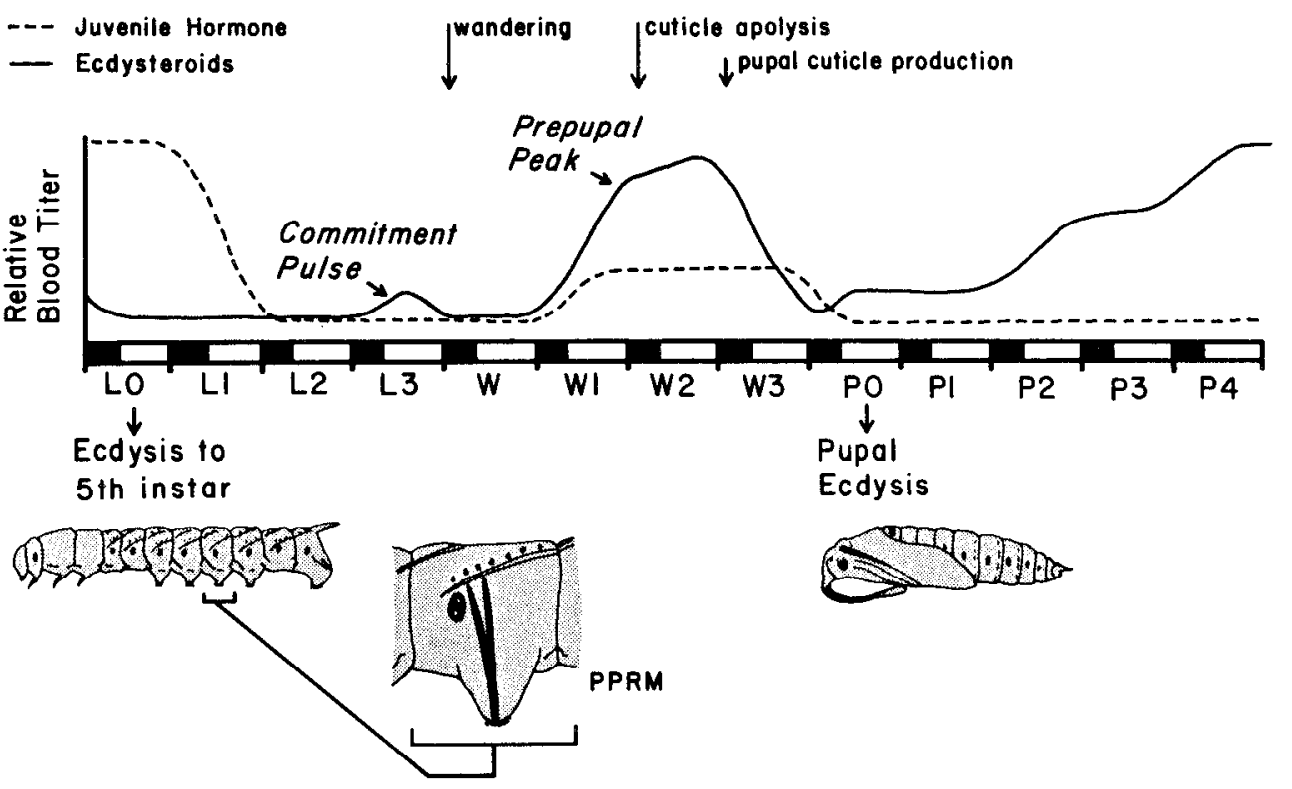

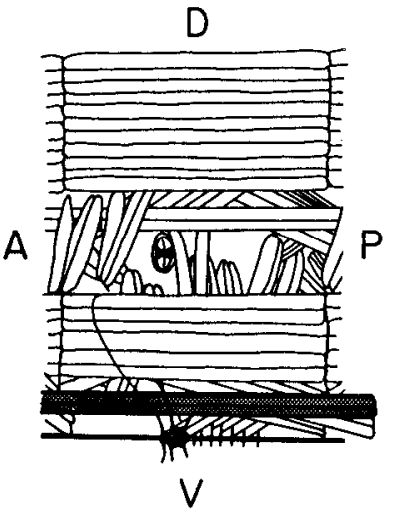

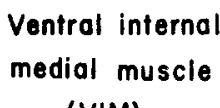

(VIM)
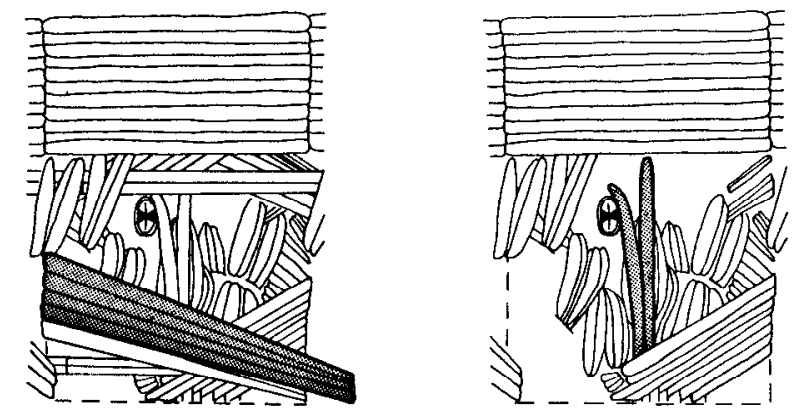

overlying larval cuticle (termed apolysis) and to produce the new pupal cuticle. Al the same lime internal organs undergo pupal development (see below). Juvenile hormone reappears during the prepupal peak to prevent precocious development of adult structures (Kiguchi and Riddiford, 1978). The fall of the prepupal peak is followed by ecdysis to the pupa, after which the ecdysteroid titer rises again to promote adult development.

The transformation to the pupa is accompanied by loss of function in the abdominal prolegs (Fig. 1). The prolegs are controlled by a set of retractor muscles, the largest of which is PPRM (Fig. 1, inset; Weeks and Truman, 1984b). This muscle consists of two muscle fibers which insert at separate points on the dorsal body wall and run to the tip of the proleg, the planta. PPRM's contraction causes the crochets (sclerotized hooks on the planta) to be disengaged from the substrate and the proleg to be retracted toward the body. The prolegs participate in many larval behaviors such as crawling and ecdysis (Weeks and Truman, 1984a, b). They are used for the final time during wandering behavior, after which the body cuticle stittens and the prolegs harden and flatten toward the body. After day $\mathrm{W} 2$ proleg retractions can no longer be evoked. The role of blood-borne ecdysteroids in dismantling the proleg motor system is described in the following sections.

\section{Principal planta \\ retractor muscle (PPRM)}

\section{Ventral external \\ (VEO)}

Endocrine control of muscle degeneration

Time course of normal muscle degeneration. During the larvalpupal transformation of Manduca, all of the external muscles of the abdomen degenerate (Taylor and Truman, 1974). These muscles die in two waves; a minority degenerate prior to pupal ecdysis, whereas the majority remain normal through day PO but degenerate thereafter. The only major muscle groups remaining in the pupal abdomen are the internal intersegmental muscles, which survive until adulthood. The new external muscles of the moth differentiate from myoblasts during adult development (Stocker and Nuesch, 1975)

Figure 2 illustrates representatives of the three classes of larval muscles. PPRM has already been described (Fig. 1). VEO is an external intersegmental oblique muscle and VIM is an internal intersegmental muscle. The status of these muscles was followed by measuring their fiber diameters (see "Materials and Methods"). The diameters of PPRM's two fibers were measured near the spiracle, and these values were summed. The diameters of the most dorsal VEO fiber and the most medial VIM fiber were measured at midsegment.

The fates of these muscles during the larval-pupal transformation are shown in Figure 3. All muscle fibers attained their full size by 

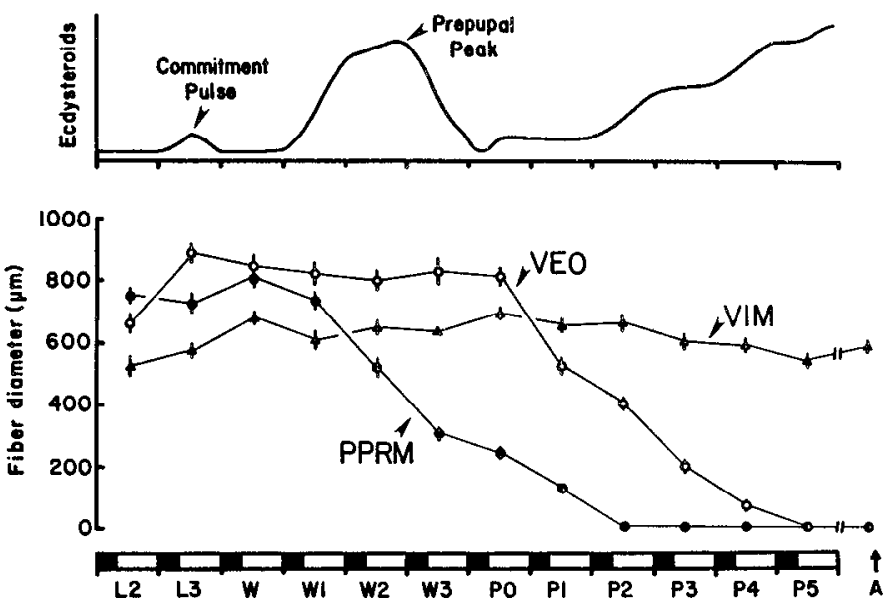

Figure 3. Muscle degeneration during the larval-pupal transformation. To $\rho$, Bloud ecdysteroid profile. Bottom, Measurements of muscle fiber diameter in muscles VEO (O), VIM (A), and PPRM (O). For VEO and VIM, each point is at least 20 muscles from three or more animals. Each PPRM point represents the sum of the two fibers in the muscle (Figs. 1 and 2), measured in at least 20 muscles from three or more animals. Error bars smaller than the symbols are not indicated. The first day on which PPRM's fiber diameter was significantly reduced was $W 2$ ( $\rho<0.0005$, one-tailed $t$ test). $A$, adult; these measurements were made just prior to adult emergence.

day $W$. VIM remained stable during pupation and survived unchariged until adult emergence, whereas the two other muscles degenerated. PPRM fiber diameter became significantly reduced on day W2. VEO remained normal through pupal ecdysis but degeneration was underway by day $\mathrm{P1}$. Both muscles took 5 days to undergo complete histolysis.

Fffects of ecdysteroid manipulations on muscle degeneration. Because ecdysteroids regulate changes in many body tissues during metamorphosis (Riddiford, 1985), we first tested whether these hormones were involved in the fates of PPRM, VEO, and VIM. The observation that PPRM began to degenerate soon after the initial rise in blood ecdysteroids during the prepupal peak (Fig. 3) suggested that this steroid increase might trigger the degeneration. If so, then prevention of the prepupal peak should rescue PPRM. This possibility was tested by ligaling larvae between the thorax and abdomen, thereby separating the abdominal muscles from the ecdysone-secreting glands in the thorax. This manipulation also eliminated the source of juvenile hormone in the head. Depending upon whether the abdomen was isolated on day $W$ or $L 2$, one or both steroid peaks could be prevented. Isolated abdomens survived for weeks and remained arrested in the developmental stage at which they were ligated.

To determine whether the effects of ligation were due to the removal of ecdysteroids, some abdomens were infused with 20-HE. As shown previously (Nijhout, 1976), post-wandering abdomens responded to infused $20-\mathrm{HE}$ by terminating their developmental arrest as indicated by cuticle apolysis, production of pupal cuticle, and, as will be shown, pupal development of the internal organs.

Figure 4 compares the fates of VIM, VEO, and PPRM in control, ligated, and ligated and infused abdomens. All abdomens were ligated on day $W$, following the commitment pulse, and the infused groups were infused for $12 \mathrm{hr}$ on day W1. VIM survived in all cases (Fig. 4A). In contrast, VEO and PPRM survived in ligated abdomens but degenerated when 20-HE was infused (Fig.4, B and C). PPRM degenerated regardless of whether the infusion occurred at the normal onset of the prepupal peak (day $W_{1}$ ) or was delayed until 5 days post-ligation (Fig. 4C). All muscles which survived the time period shown in Figure 4 were still alive when examined at later times (data not shown). Muscles which retained their larval fiber diameters also remained contractile in response to nerve stimulation or forceps pinch. In other experiments, abdomens were treated with
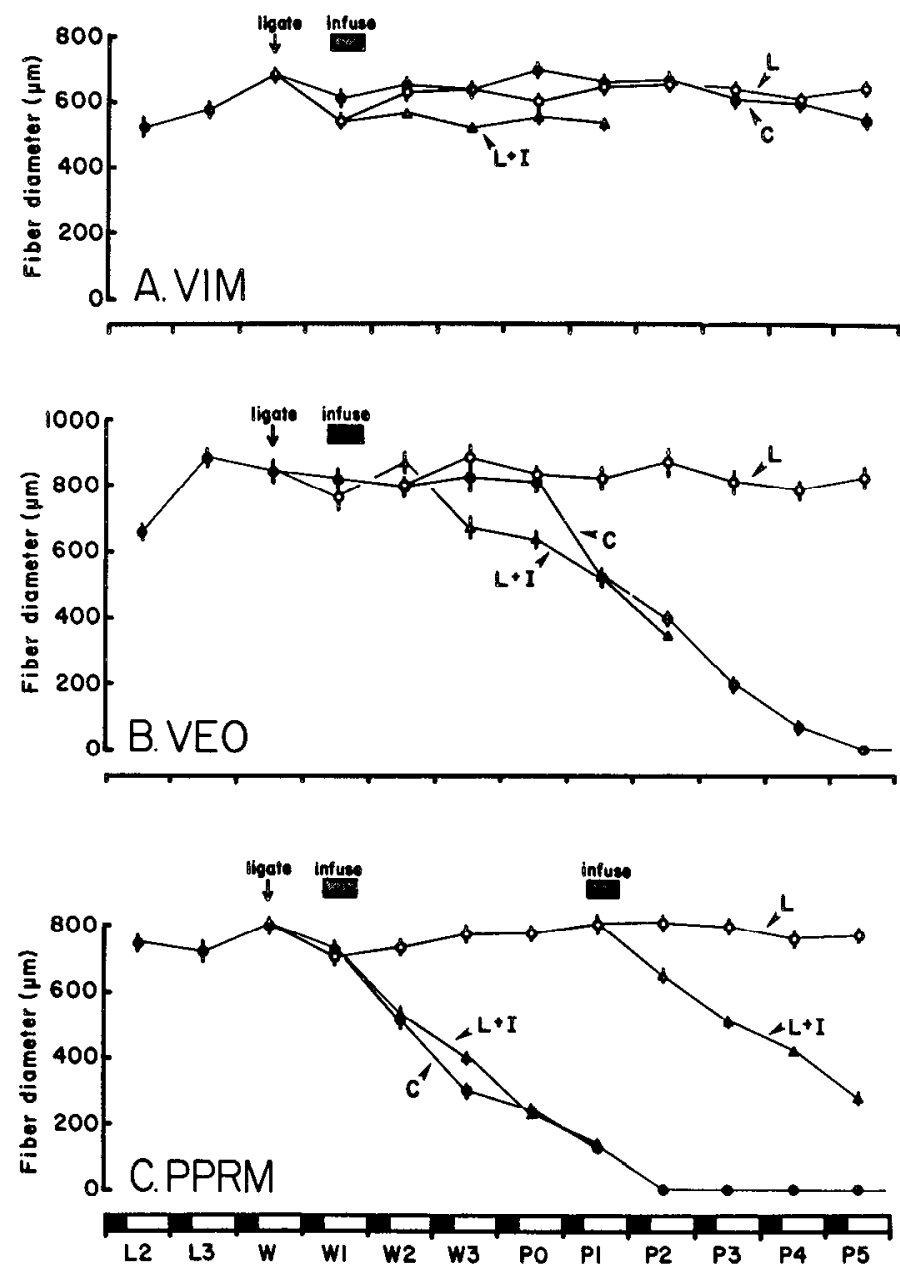

Figure 4. Effects of steroid manipulations on the fates of larval muscles. $A, B$, and $C$ present muscle fiber diameter measurements made in control $(C ; \mathbf{O})$, abdomen ligated $(L ; O)$, or ligated and infused $(L+l ; \mathbf{A})$ preparations The control data are the same as those in Figure 3 . All abdomen ligations were performed on day $W$ (arrows). Ligated abdomens were either held and fiber measurements made up to 9 days later $(L)$, or else the abdomens were infused with $20-\mathrm{HE}$ on day $W 1$ (shaded boxes), with measurements made over the following 4 days $(L+1)$. For PPRM, some animals were ligated and then held for 5 days before infusion $(C$, second shaded box). All infusions were $5.6 \mu \mathrm{g}$ of $20-\mathrm{HE} / \mathrm{hr}$ for $12 \mathrm{hr}$. Each point is the mean ( $\pm \mathrm{SEM}$ ) fiber diameter from at least 20 muscles in three or more animals.

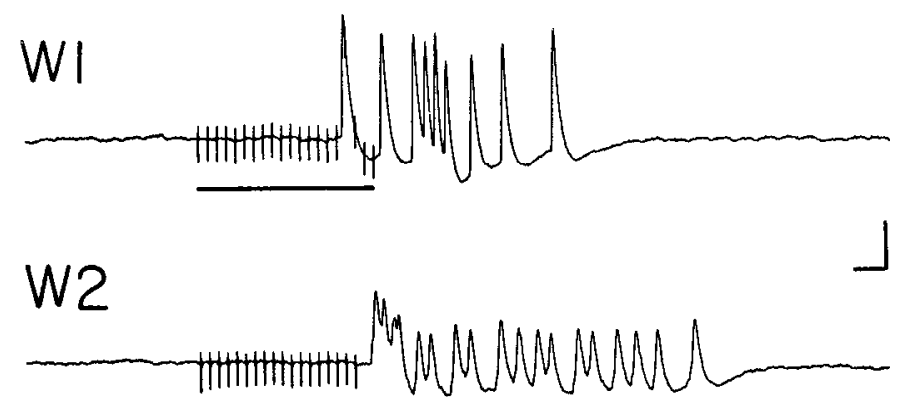

Figure 5. Electrical responsiveness of PPRM during its degeneration. Each trace is an extracellular recording of a PPRM fiber made on day $\mathrm{W}_{1}$ (top) or W2 (bottom). During each bar a train of shocks indicated by the stimulus artifacts (approximately $30-\mathrm{Hz}, 500-\mathrm{msec}$ duration pulses in both cases) was delivered via a suction electrode to the $\mathrm{VN}$ contralateral to the recorded muscle. Scale bars $=100 \mathrm{msec}, 1 \mathrm{mV}$; the recording technique shows only relative sizes of the responses. 
TABLE I

Effects of denervation and ecdysteroid infusion on PPRM's degeneration

\begin{tabular}{|c|c|c|c|}
\hline \multirow{2}{*}{ Treatment } & \multirow{2}{*}{$N^{a}$} & \multicolumn{2}{|c|}{ PPRM Fiber Diameter $(\mu \mathrm{m})$} \\
\hline & & Denervated & Control \\
\hline & & \multicolumn{2}{|c|}{ Mean \pm SEM } \\
\hline $\operatorname{Vn} x^{b}$ and ligation on day $L 2^{c}$ & 13 & $847 \pm 30$ & $754 \pm 43$ \\
\hline VNx and ligation on day $W^{c}$ & 10 & $750 \pm 42$ & $781 \pm 55$ \\
\hline $\begin{array}{l}\text { VNx and ligation on day } W \text {, fol- } \\
\text { lowed by } 20-\mathrm{HE} \text { infusion }\end{array}$ & 9 & $363 \pm 54^{e}$ & $385 \pm 50^{e}$ \\
\hline
\end{tabular}

${ }^{a} N$, number of paired muscles, each pair in a different abdomen.

${ }^{b} \mathrm{VNx}$, severance of the ventral nerve to denervate PPRM.

- Fibers were measured 7 days after treatment.

${ }^{a}$ Infusion $(5.6 \mu \mathrm{g} 20-\mathrm{HE} / \mathrm{hr}$ for $12 \mathrm{hr}$ ) was given 5 days post-ligation; fibers were measured 2 to 3 days post-infusion.

e Value is significantly smaller ( $p<0.0005$, one-tailed $t$ test) than the value on the line immediately above.

a juvenile hormone analogue during the infusion to mimic the normal co-occurrence of these hormones during the prepupal peak (Fig. 1). This had no effect on the pattern of muscle degeneration (J. C. Weeks, unpublished observation)

These data show that prevention of the prepupal ecdysteroid peak blocked the degeneration of larval muscles. The commitment pulse was by itself insufficient to trigger degeneration, since all muscles survived in abdomens isolated on day $W$. However, infusion of $20-\mathrm{HE}$ into these abdomens triggered the appropriate pattern of muscle-specific degeneration, and this response was independent of the presence or absence of juvenile hormone.

Timing of early and late waves of muscle degeneration. In intact animals, PPRM began to degenerate during the rising phase of the prepupal peak. The later onset of degeneration in VEO (Fig. 3) suggested that a different signal, perhaps the falling phase of the prepupal peak, might trigger its death. If so, this would explain why VEO degenerated somewhat earlier in infused abdomens than in intact animals ( $\mathrm{Fig}$. 4B), since the abdomens experienced a 12-hr steroid infusion rather than the normal 48-hr exposure (Fig. 1). This effect was not attributable to juvenile hormone, because in infused abdomens treated with juvenile hormone, VEO degenerated with the same time course as in Figure 4 (data not shown). To determine whether the time of the fall in the steroid titer was important, abdomens were isolated on day $W$ and infused on day $W+$ with a 36-hr infusion rather than the usual 12-hr treatment (20-HE was delivered at the same rate in both cases). Muscle fibers were measured approximately $48 \mathrm{hr}$ after the start of the infusions. If VEO'S degeneration was initiated by the fall in ecdysteroids, then muscles in the 12-hr group should have been more advanced in their degeneration than muscles in the 36-hr group. Instead, degeneration was the sarne in both groups; VEO's fiber diarneter was 642 $\pm 31 \mu \mathrm{m}$ after the 12-hr infusion (data from Fig. 4B) and $661 \pm 23$ $\mu \mathrm{m}$ after the $36-\mathrm{hr}$ infusion (mean $\pm \mathrm{SEM}, N>20$ fibers from at least four abdomens). PPRM and VIM were similarly unaffected by the length of the infusion (data not shown). These findings do not support the hypothesis that the fall of ecdysteroids triggers VEO's degeneration.

In another set of experiments, intact animals were infused continuously for 5 days beginning on day W2 with $5.6,11.2$, or $16.8 \mu \mathrm{g}$ of $20-\mathrm{HE} / \mathrm{hr}$. This infusion should have kept blood ecdysteroids elevated past the normal fall of the prepupal peak. Infused animals showed a delay of approximately 1 day in their ecdyses, similar to that caused by injections of 20-HE late in the prepupal stage (Truman et al., 1983). These animals also showed a commensurate delay of about 1 day in the degeneration of their VEO's (data not shown). Thus, prolonged elevation of ecdysteroid levels in intact animals can delay the terminal events in the larval-pupal transformation, including the late wave of muscle degeneration. This effect was not observed in isolated abdomens, at least under the 20-HE infusion paradigms described above. Additional experiments are needed to determine how the timing of the late wave of muscle degeneration is controlled.

Role of neuromuscular interactions in muscle degeneration. Blood-borne ecdysteroids could cause PPRM and VEO to degenerate by acting directly on the muscles, or else via indirect mechanisms such as causing neural input to be withdrawn. The latter possibility was examined in PPRM by testing neuromuscular transmission in normal animals before and during degeneration. One VN in a segment was stimulated electrically to activate a bilateral proleg retraction reflex (Weeks and Truman, 1984b), while recording from the contralateral PPRM. This stimulus evoked electrical responses and contractions in PPRM on days W1 and W2 (Fig. 5). The recorded events, which are assumed to be graded action potentials (Dietmer, 1977), became progressively smaller as pupation neared. Weak responses were still present on day $W 3$, but by pupal ecdysis the muscle was unresponsive to either VN stimulation or forceps pinch (data not shown). Thus, PPRM was driven by its motoneuron in segmental reflexes even during the terminal stages of its degeneration.

PPR's possible influence on PPRM's degeneration was further examined by denervating the muscle. Abdomens were isolated on either day $L 2$ or $W$, and the $V N$ in one abdominal hemisegment was severed surgically. This paradigm allowed the effect of denervation to be examined in the absence of circulating ecdysteroids. One week after surgery, the fiber diameters of muscles denervated on days $L 2$ or $W$ were no smaller than those of the contralateral, normally innervated muscle (Table I), and they contracted normally when pinched. The status of PPRM's distal severed axon was not examined, but based on other studies, it was expected to undergo degeneration within a few days (Nuesch and Stocker, 1975).

To see whether denervated muscles could still degenerate in response to infused steroid, PPRM denervation and abdomen isolation were performed on day $W$, and 5 days later the abdomens were infused for $12 \mathrm{hr}$ with 20-HE. Muscle fibers were measured 2 to 3 days post-infusion. Both the denervated muscles and their normally innervated homologues degenerated normally in response to the infusion (Table I). Thus, the presence or absence of PPR's innervation is irrelevant to PPRM's ecdysteroid-mediated degeneration.

\section{Endocrine control of dendritic reduction and death in motoneuron PPR}

Time course of dendritic reduction during metamorphosis. Changes in PPR's morphology during the larval-pupal transformation were examined in cobalt-stained neurons. After PPRM became nonfunctional, PPR could not be identified during intracellular penetrations by its motor effects. However, after staining, the motoneuron could be unambiguously identified by its unique bilateral arbor (Fig. 6). PPR's structure was also examined in ganglia in which the lateral branch of the $\mathrm{VN}\left(\mathrm{VN}_{\mathrm{I}}\right)$ was backfilled with cobalt. In the prolegbearing segments of larvae, seven motoneurons run in this nerve; all have ipsilateral cell bodies and arborize in the ipsilateral hemineuropil, but only PPR crosses the midline to produce a contralateral arbor (Weeks and Truman, 1984b). This portion of its arbor can thereby be viewed in isolation.

Both intracellular fills (Fig. 6, $A$ and $B$ ) and backfills (Fig. 6, $D$ and $E$ ) revealed that PPR's arbor regressed substantially during the larval-pupal transformation. This regression occurred in both the ipsiand contralateral arbor and resulted in a decrease in the total volume of neuropil to which PPR projected. Particularly striking was a reduction in the number of higher-order branches and spines on PPR's processes. These structural changes were analyzed using a morphometric system which measured PPR's "dendritic density" (see "Materials and Methods"). The time course of PPR's regression is shown in Figure 7. The neuron tirst became reduced on day $\mathrm{W} 1$, and by day P1 its dendritic density was $40 \%$ lower than its initial value. Starting on day $\mathrm{P} 2$, backfills of $V N_{1}$ filled six rather than seven neurons (with the missing cell body located in PPR's position), and 

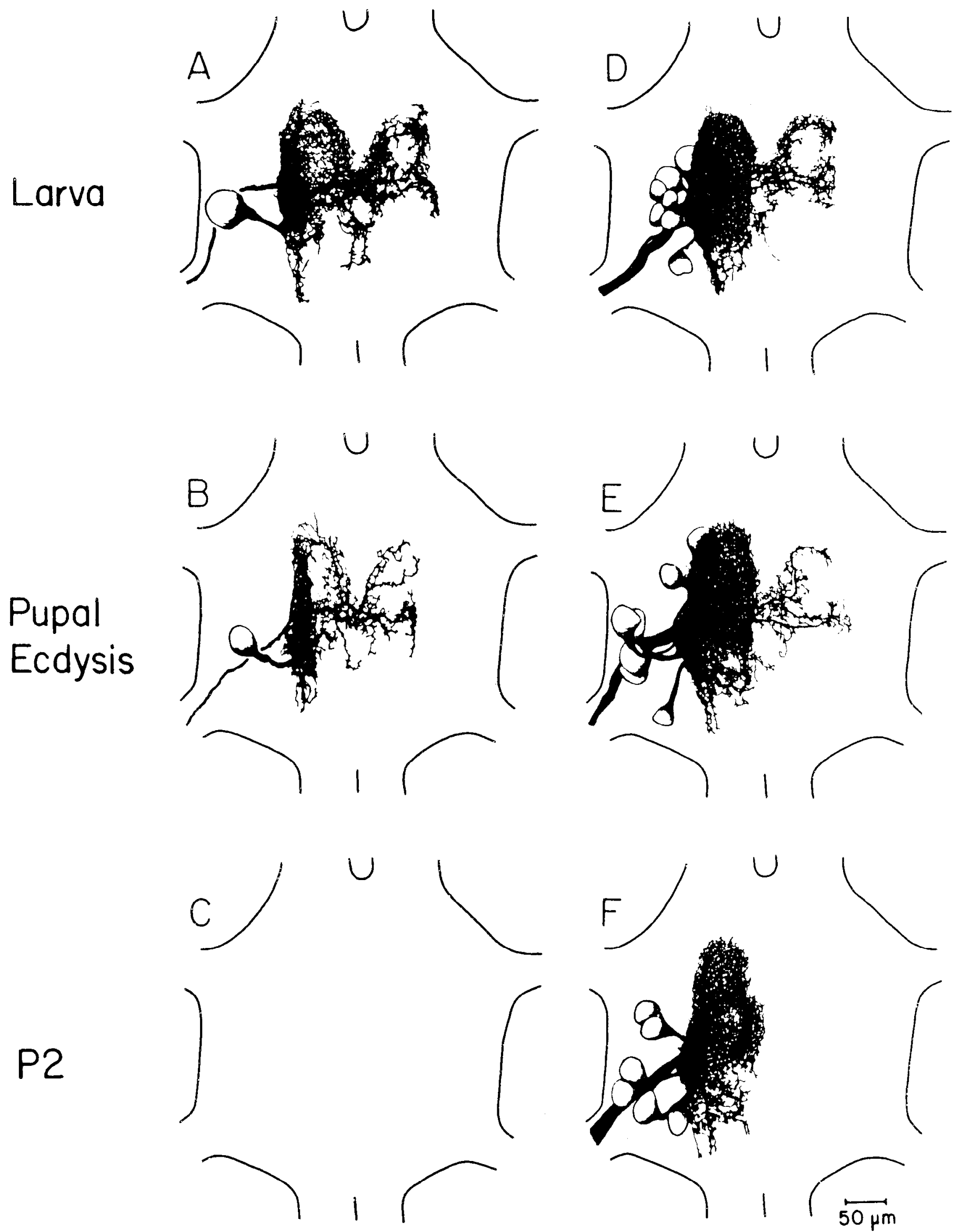

Figure 6. Morphology of PPR during the larval-pupal transformation. The left column shows intracellular cobalt fills of PPR; the right column shows cobalt backfills of $V N_{1}$ made in larvae $(A$ and $D)$, at pupal ecdysis $(B$ and $E$ ), and 2 days after pupal ecdysis $(C$ and $F$ ). Camera lucida drawings were made from ganglia in whole mount, viewed trom the dorsal aspect. Backtills of VN in larvae stain seven neurons, of which two have ventral and five (including PPR) have dorsal cell bodies (Weeks and Truman, 1984b). Beginning on day P2 the dorsal cluster contains only four cells, indicating PPR's death 
Figure 7. Time course of dendritic reduction in PPR during the larval-pupal transformation. Each bar indicates the mean \pm SEM of PPR's dendritic density ( $N=5$ neurons analyzed per day). The dashed line indicates density prior to dendritic regression. *, first value significantly lower than previous values ( $p<0.0025$, one tailed $t$ tcst). PPR dies on day $\mathrm{P} 2$.

Figure 8. Effect of steroid manipulations on dendritic reduction in PPR. Top, Blood ecdysteroid profilc. Bottom, Camera lucida draw ings of PPR's contralateral arbor as visualized by cobalt backfilling of $V N_{1}$ in each of five experirnental cases ( $A$ to $E$ ). Scale bar $=25$ $\mu \mathrm{m}$. Numbers below each drawing are the dendritic density values in each case (mean $\pm \operatorname{SEM} ; N=5$ ). $A$ and $E$ show fills from normal L2 and PO animals (data from Fig. 7) The mean number of days between ligation and backfilling is indicated in $B$ and $C$. Den dritic densities in $A, B$, and $C$ are not significantly different. The 20-HE infusion paradigm in $D$ is the same as for Figure 4. The dendritic density in $D$ is significantly lower than that in $C$ at the $p<0.0005$ level (one-tailed $t$ test) Dendritic densities in $D$ and $E$ are not signifcantly different.

Figure 9. Effect of removal of PPRM (PPRMX) on dendritic reduction in PPR. The format of this figure is the same as in Figure 8. Scale bar $=25 \mu \mathrm{m}$. Fills in $B, C$, and $D$ are motoneurons from which target muscles were previously removed. Dendritic densities in $A$, $B$, and $C$ are not significantly different. Dendritic density in $D$ is significantly lower than that in $C$ at the $D<0.005$ level (one-tailed $t$ test). Densities in $D$ and $E$ are the same.

PPR's characteristic contralateral arbor was absent (Fig. 6F; PPR was missing in eight of nine backfills of day $P 2$ pupae). Furthermore at this time a degenerating cell body in PPR's location appeared in sectioned histological material (J. W. Truman, unpublished observations). These features have been found to indicate the death of neurons (e.g., Taylor and Truman, 1974; Truman, 1983; Giebultowicz and Iruman, 1984), and consequently we infer that PPR dies on day $\mathrm{P} 2$.

Role of ecdysteroids in PPR's dendritic regression and death PPR's structure was examined after a series of ligation and infusion experiments similar to those described above (Fig. 8). One group of abdomens was isolated on $L 2$ (prior to either steroid peak) and examined 1 to 2 weeks later, at which time the PPRs were still alive and had the same dendritic density as in normal larvae (compare Fig. $8, A$ and $B$ ). Similarly, when abdomens were isolated on day $W$ and held for 1 to 2 weeks, the PPRs retained their larval morphology (Fig. $8 C$ ). PPR's dendritic reduction and death are thus triggered by some event (or events) that occurs after day $W$.

Infusion of $20-\mathrm{HE}$ into abdomens isolated on day $W$ caused PPR to regress. By 3 days post-infusion, on the day which would normally correspond to pupal ecdysis, the dendritic density of PPRs in ligated and infused abdorneris (Fig. 8D) was virtually identical to that of day $\mathrm{PO}$ animals which experienced their endogenous prepupal peak (Fig $8 E$ ). Similar results were obtained when abdomens were treated with a juvenile hormone analogue during 20-HE infusion ( $\mathrm{J} . \mathrm{C}$. Weeks, unpublished observation). Thus, dendritic regression can be attributed to the appearance of ecdysteroids during the prepupal peak, and 20 -HE infusion adequately mimics the endogenous peak in inducing regression.

Some backfills were made at longer post-infusion intervals to determine whether PPR died. These experiments were not entirely satisfactory because the isolated abdomens were unable to shed the old larval skin and tracheal linings after producing a pupal cuticle, and beginning about 4 days post-infusion, most became moribund. In those abdomens still alive at 5 days post-infusion (which would correspond to day P2), $20 \%$ of PPRs had died ( 2 of 10 fills made in five abdomens). Six days post-infusion, $43 \%$ had died ( 6 of 14 fills in seven abdomens). In all cases, the $\mathrm{VN}$ motoneurons other than PPR were still alive. As shown above (Fig. $8 \mathrm{C}$ ), in the absence of infusion, PPR survives indefinitely in isolated abdomens. Thus, 20 $\mathrm{HE}$ infusion was able to trigger PPR's death, although in isolated abdomens this process was retarded when compared to intact animals. PPR's death was similarly delayed in abdomens which were isolated on days W3 or P0 near the end of the animal's own prepupal peak; PPR subsequently died, but 2 to 3 days later than usual (data not shown). These abdomens were also unable to shed their old cuticles.

Although the prepupal ecdysteroid peak was somehow responsible for PPR's death, it is not clear whether the commitment to die was made at the same time as the commitment to undergo dendritic regression. Since post-emergence neuronal death in adult Manduca is triggered by a fall in blood ecdysteroids (Truman and Schwartz, 1984), we investigated the possibility that PPR's death was caused by the fall in the prepupal peak. In one set of experiments we made single injections of $20-\mathrm{Ht}(25$ or $50 \mu \mathrm{g})$ into animals at different times during the $12 \mathrm{hr}$ preceding pupal ecdysis, when the steroid titer is normally falling. This treatment, which delays some of the terminal events of pupal development (Truman et al., 1983), occasionally delayed PPR's death by a day but not reproducibly (data not shown). Io produce a more physiological elevation of blood steroids we infused intact animals continuously for 5 days beginning on $\mathrm{W}_{2}$ (at $5.6,11.2$ or $16.8 \mu \mathrm{g} / \mathrm{hr}$, same paradigm as described previously for VEO measurements). VN backfills were made 3 days after pupal ecdysis (which was delayed by a day by the infusions), and in all cases, PPR had died ( $N=9$ fills in seven animals). Thus, using these paradigms we were unable to attribute PPR's death specifically to the falling phase of the prepupal peak.

Relationship of PPRM's degeneration to dendritic regression and death in PPR. The above experiments indicated that the prepupal ecdysteroid peak is somehow responsible for triggering PPR's dendritic regression and death. Since regression is normally underway on day $\mathrm{W}_{1}$ (Fig. 7), it must bc initiatcd by the rising phase of the prepupal peak. This signal also triggers PPRM's degeneration (Fig. 4). Accordingly, the infusion experiments could not distinguish whether PPR's dendritic regression or death resulted from a steroid action on the nervous system or, instead, were indirectly caused by the concomitant degeneration of PPRM.

Since PPR made functional neuromuscular contact with PPRM through day $W 3$, it was possible that some interaction with its dying muscle caused PPR to regress or to die. To test this, we examined the effects of surgically removing PPRM (PPRMx). Measurements made in PPRMx and control abdominal segments indicated that this technique removed an average of $89 \%$ of the muscle's length $(N=$ 14 muscle pairs measured), leaving only small remnants at the dorsal insertion points of the fibers. Anatomical studies in methylene bluestained control preparations showed that nerve branches from VN joined the muscle only near its ventral end. In operated segments these branches were usually broken at the point at which they had contacted the fibers. This technique thus eliminated PPR's contact with PPRM by causing axotomy of the motoneuron very near the muscle.

As seen in Figure 9, $B$ and $C$, PPRMx and abdomen isolation on day $W$ or $L 2$ did not affect PPR's dendritic density when the targetless motoneuron was examined 7 days later. Thus, loss of synaplic conlact with its target muscle is not by itself sufficient to cause PPR to reduce its arbor or to die. However, it might be argued that PPR's loss of contact with PPRM by distal axotomy did not provide the same signal as might normally be provided by maintained contact with a degenerating muscle. The possibility that PPR required an interaction with its muscle in order to regress was ruled out by the experiment in Figure 9D. After ligation and PPRMx on day $W$, abdomens were held for 4 days and then infused with 20 $\mathrm{HE}$. As was shown in Figure $4 \mathrm{C}$, isolated abdomens retained their steroid sensitivity and responded to a delayed infusion by proceeding with pupal development. When filled 3 days post-infusion, both the targetless motoneurons (Fig. 9D) and motoneurons in which muscles had not been removed (data not shown) had regressed to the extent expected for new pupae (Fig. 9E). Thus, PPR responded to the ecdysteroids despite the fact that it was not in contact with its target muscle during the time the hormones acted.

To determine whether PPR would die at the usual time even if the motoneuron was prematurely rendered targetless, a PPRM was removed from day $L 0$ intact larvae. These larvae grew to a mature size and initiated metamorphosis at the normal time. On day P1, $100 \%$ of the targetless PPRs wcre still alive and had the expected reduced arbor ( $N=3$ animals), whereas on P2 the targetless PPRs were all dead ( $N=3$ animals). Thus, elimination of PPRM for the entire fifth instar did not alter PPR's fate.

\section{Discussion}

The phenomena observed in the proleg system-muscle degeneration, dendritic regression, and neuronal death-occur during the development of many animals. For instance, the regression of neurites or dendrites occurs during development of vertebrates (Falls and Gobel, 1979), other insects (Goodman et al., 1981), and other invertebrates (Wallace, 1984). In Manduca, the wealth of available information regarding the endocrinology of metamorphosis has allowed us to test specifically the role of blood-borne steroids in controlling the fates of neurons and muscles. We found, in contrast to some systems (e.g., Hamburger and Oppenheim, 1982), that interactions between neurons and muscles were not of paramount importance. Instead, their development is coordinated by endocrine cues acting independently at the two sites. This is not an exclusively invertebrate attribute, since independent steroid actions on motoneurons and their muscles have also been reported during vertebrate development (Breedlove and Arnold, 1983). Certainly, further com- 
parative studies are needed to fully reveal how blood-borne steroids and cellular interactions contribute to neuromuscular development.

Ecdysteroid-mediated muscle degeneration. At pupation, larval muscles fall into three differently fated classes as represented by VIM, VEO, and PPRM. VIM in mid-abdominal segments is indifferent to ecdysteroids at this time and persists regardless of the presence or absence of steroids, although the muscle eventually dies in response to the fall in blood steroids at the completion of adult development (Schwartz and Truman, 1983). Unlike VIM, muscles PPRM and VEO die during the larval-pupal transformation. The early wave of muscle death includes PPRM and accompanies the elimination of the prolegs as the pupal body is formed. The later, more extensive wave of muscle death follows pupal ecdysis, a behavior in which many of the late-dying muscles such as VEO participate (Weeks and Truman, 1984a).

As indicated by ligation and infusion experiments, the degeneration of PPHM and VEO is normally triggered by the prepupal ecdysteroid peak. Although juvenile hormone normally reappears during the peak, it need not be present in the blood in order for degeneration to occur. The degeneration of PPRM (and presumably of the other early-dying muscles) is apparently initiated by the upswing of the prepupal ecdysteroid peak because histolysis begins while the steroid titer still is rising. The regulation of the death of latedying muscles such as VEO is more perplexing. In infused abdomens and intact animals, VEO can begin degenerating without having seen a drop in ecdysteroids, suggesting that something associated with the rise or plateau of the prepupal peak initiates degeneration. Yet in the prepupae infused for 5 days, the elevated steroid titer delayed the terminal events of pupal development, including VEO's degeneration, by about a day. Thus, the regulation of muscle death after pupal ecdysis is not as simple as that after adult ecdysis when degeneralion is triggered solely by the withdrawal of blood steroids (Schwartz and Truman, 1983). Preliminary experiments suggested that the presence or absence of juvenile hormone during the prepupal peak does not influence the timing of muscle degeneration (J. C. Weeks, unpublished observations). Further experiments are needed to clarify this situation.

At least as reflected in fiber diameter measurements, muscles do not respond overtly to the commitment pulse of ecdysteroids, and in abdomens ligated on the day after the pulse the muscles persist indefinitely. By analogy to other tissues, however, the appearance of ecdysteroids in the absence of juvenile hormone during the commitment pulse may initiate changes in muscle mRNA and protein synthesis which set the stage for later responses during the prepupal peak (e.g., Riddiford, 1981). Yet in experiments not presented here (J. C. Weeks, unpublished observations), infusion of the usual 12-hr dose of 20 - HE into abdomens isolated on day $L 2$ caused PPRM and VEO to degenerate in their proper sequence, although more slowly than usual. Thus, even though degeneration is normally evoked by the second ecdysteroid peak, a single large ecdysteroid exposure is sufficient to elicit a pupal developmental program in the muscles. This is not true of all tissues (see below).

PPRM's fate is not influenced by interactions with its motoneuron. The muscle continued to be driven by PPR even during the terminal stages of its degeneration. Furthermore, chronic denervation of PPRM did not cause degeneration, but denervated muscles degenerated in tandem with their innervated homologues when exposed to the proper endocrine regimen. Thus, loss of neural input is not by itself sufficient to cause histolysis, and innervation need not be present for PPRM to show steroid-induced degeneration.

The fate of a similar proleg muscle has been studied in the greater wax moth Galleria mellonella (Randall, 1968, 1970; Randall and Pipa, 1969; Runion and Pipa, 1970). Unlike PPRM in Manduca, the muscle in Galleria degenerates following pupal ecdysis. Runion and Pipa (1970) found that the muscle continued to be driven neurally during its degeneration and that chronic electrical stimulation could not prevent degeneration. Randall (1970) found that when denervation and abdomen isolation were performed prior to the onset of meta- morphosis, the muscle survived indefinitely, although some atrophy was apparent at the ultrastructural level. Randall (1970) also found that injection of $20-\mathrm{HE}$ caused degenerative changes, even in denervated muscles. These investigators concluded that proleg muscle degeneration in Galleria was governed by endocrine rather than neural factors.

The endocrine control of muscle degeneration is not unique to the proleg system. The post-emergence degeneration of the intersegmental muscles has been studicd in a number of lepidopteran species (e.g., Finlayson, 1956; Lockshin and Williams, 1965; Schwartz and Truman, 1983, 1984), and the principal finding has been that muscle degeneration is hormonally triggered independently of neural input. It has also been shown that muscles destined to die during metamorphosis will do so in organ culture in response to ecdysteroids (Zachary and Hoffmann, 1980).

Ecdysteroid control of motoneuron structure and survival. The programmed degeneration of the proleg muscles during the larvalpupal transformation renders their motoneurons targetless. Levine and Truman $(1982,1985)$ studied a number of motoneurons with axons in the dorsal nerve which lose their muscles at pupation, and found that these cells subsequently have two options. The majority survive the death of their muscles and become respecified to innervate new adult muscles. The remainder of the targetless motoneurons die on day $\mathrm{P} 2$, which is the only major period of motoneuron death during the larval-pupal transformation (Giebultowicz and Truman, 1984; J. W. Truman, unpublished observations). Thus there are two waves of muscle death but only one wave of motoneuron death. When examined on day $\mathrm{PO}$, none of the motoneurons supplying late-dying muscles (for instance, $\mathrm{MN}-6$, or the motoneuron of muscle VEO) have reduced arbors (Weeks and Truman, 1984a; J. C. Weeks, unpublished observations). In contrast, motoneurons wilh muscles that have died by this time (e.g., PPR and APR; Weeks and Truman, 1984b) are significantly reduced by day $P O$. This observation was the initial impetus which led us to question whether it was the ongoing degeneration of their target muscles that caused the proleg motoneurons to regress.

Although we found that PPRM's degeneration was independent of PPR, the opposite could not be assumed automatically. At the same time that PPR begins its transformation, the ecdysteroid titers are changing and PPRM is beginning to degenerate. However, interactions with PPRM do not contribute to PPR's regression and death. Surgical elimination of PPRM had no effect on PPR, and 20HE infusion elicited PPR's regression and death regardless of whether the motoneuron was in contact with its muscle. The finding that PPR's death is not tied to the death of its muscle is perhaps not surprising, given that most Manduca motoneurons having target musclos which dic at pupation survive. The independence of motoneuron and muscle death has also been demonstrated for the postemergence period in adult Manduca (Truman and Schwartz, 1983). Similarly, muscle removal does not affect the differentiation of motoneurons during embryonic development in the grasshopper (Whitington et al., 1982). The rolc of target muscles in determining the structure and survival of motoneurons has also been studied in vertebrate systems. Ablation of target muscles has often been shown to cause enhanced motoneuron death during development (Oppenheim et al., 1978), and even in mature animals, motoneuron arbors can be induced to regress by climination of ncuromuscular contact (Sumner and Watson, 1971). Further studies need to be carried out to determine whether such findings reflect a fundamental difference between vertebrates and invertebrates, or simply reflect the limited number of systems which have been examined.

The elimination of PPRM as the determinant of PPR's regrossion and death suggest that ecdysteroids control these events by acting directly on the CNS rather than indirectly via the periphery. PPR does not respond overtly to the commitment pulse, but a subsequent ecdysteroid exposure, whether of endogenous origin (in intact animals) or provided by infusion (in isolated abdomens), elicits dendritic regression and death. Juvenile hormone did not have to be present 
in order for PPR to show normal pupal responses. PPR's regression begins while the prepupal peak is still rising, and although the prepupal peak also leads to PPR's death, it is less clear which aspect of the peak is responsible for the latter event. One possibility is that the rise in steroids initiates both dendritic reduction and a program of cell death, but that the two processes have different latencies. This mechanism has been proposed to control the timing of neuronal death after emergence of the adult moth (Bennett and Truman, 1985). Because post-emergence neuron death in Manduca is triggered by a decline in blood steroids (Truman and Schwartz, 1983), we tested whether the falling phase of the prepupal peak caused PPR to die but found no consistent support for this possibility. However, even if we cannot presently pinpoint the aspect of the steroid profile that commits PPR to dic, there is good rcason to believe that dendritic regression and death are programmed separately (see below).

To investigate whether the commitment pulse plays an essential role during development, PPR was stained in abdomens which were isolated on day $L 2$ and infused with $20-H E$ (J. C. Weeks, unpublished observations). As discussed above, such abdomens showed normal but somewhat slower muscle degeneration. In contrast, PPR did not respond normally; 3 days post-infusion, the motoneuron was still in its larval state, and after 7 days, almost all of the motoneurons were still alive and their mean dendritic density had decreased to only about 0.30 (equivalent to a day $W 2$ or $W 3$ animal; Fig. 7). The same infusion given to post-wandering abdomens causes substantial dendritic regression by 3 days post-infusion and PPR subsequently dies (Fig. 8D). These results suggest that the sequence of two ecdysteroid exposures may be important to PPR. Similarly, Nijhout (1976) could elicit normal pupal cuticle production in Manduca abdomens isolated on day L2 only by giving two sequential infusions. Tissues clearly differ in their steroid requirements for proper larval-pupal transformation.

The finding that most PPRs regressed but did not die in these abdomens suggests that the two processes are separable. This result would not be expected if dendritic regression were simply a symptom of impending death. The independence of these events is perhaps most strongly supported by observations from another proleg motoneuron, APR. APR's target muscle degenerates simultaneously with PPRM, and APR regresses similarly to PPR (Weeks and Truman, 1984b). However, APR does not die after pupation and instead survives until adulthood, presumably innervating a new adult muscle. These findings indicate that dendritic regression and death are independent developmental options. Thus, PPR exhibits both phenomena, APR exhibits regression but not death, and other motoneurons such as MN-6 and the motoneuron of VEO die without first regressing (J. C. Weeks, unpublished observations).

The present results support a direct action of ecdysteroids on the nervous system in controlling PPR's fate, in parallel with and independent of their effects in the periphery. The ability of ecdysteroids to act directly on the CNS to elicit programmed motoneuron death at adull emergence in Manduca has now been shown definitively using abdominal ganglia in organ culture (Bennett and Truman, 1985). However, as has been discussed by Arnold and Gorski (1984), given a steroid effect on the nervous system one must ultimately determine which neurons are direct hormonal targets and which neurons are indirectly affected. It is generally accepted that neuronal death can be mediated by intrinsic programs, which may be activated hormonally (reviewed in Truman, 1984). It is not unreasonable to assume that PPR dies by this direct mechanism. But it is less clear how dendritic regression might come about. One plausible indired rrechanism would be if other neurons were hormonally induced to withdraw their synaptic contacts onto PPR (and APR), thereby deafferenting them. The deafferentation of neurons can cause dendritic arbors to be stunted during development (Murphey et al., 1975) and can cause arbors to regress in mature animals (Jones and Thomas, 1962). PPR and APR receive some synaptic inputs even on day PO (Weeks and Truman, 1984b), but the question of deafferentation needs to be addressed by identifying neurons directly presynaptic to the larval proleg motoneurons, and following the status of these synapses throughout the larval-pupal transformation. Another unanswered question that such studies might answer is what physiological function dendritic regression serves. A determination of the consequences of dendritic regression for the motoneurons' participation in neuronal circuits will further elucidate the role played by ecdysteroids in coordinating the diverse anatomical and behavioral changes that occur during metamorphosis.

\section{References}

Arnold, A. P., and R. A. Gorski (1984) Gonadal steroid induction of structural sex differences in the central nervous system. Annu. Rev. Neurosci. 7: 413-442.

Bacon, J. P., and J. S. Altman (1977) A silver intensification method for cobalt filled neurons in whole mount preparations. Brain Res. 138: 359363.

Beckage, N. E., and L. M. Riddiford (1982) Effects of parasitism by Apanteles congregatus on the endocrine physiology of the tobacco hornworm, Manduca sexta. Gen. Comp. Endocrinol. 47: 308-322.

Bell, R. A., and F. A. Joachim (1978) Techniques for rearing laboratory colonies of tobacco hornworms and pink bollworms. Ann. Entomol. Soc. Am. 69: 365-373.

Bennett, K., and J. W. Truman (1985) Steroid-dependent survival of identifiable neurons in cultured ganglia of the moth Manduca sexta. Science, in press.

Bollenbacher, W. E., S. L. Smith, W. Goodman, and L. I. Gilbert (1981) Ecdysteroid titer during larval-pupal-adult development of the tobacco hornworm, Manduca sexta. Gen. Comp. Endocrinol. 44: 302-306.

Breedlove, S. M., and A. P. Arnold (1983) Hormonal control of a developing neuromuscular system. II. Sensitive periods for the androgen-induced masculinization of the rat spinal nucleus of the bulbocavernosus. J. Neurosci. 3: 424-432.

Brogan, R. T., and R. M. Pitman (1981) Axonal regeneration in an identified insect motoneurone. J. Physiol. (Lond.) 319: 34P-35P.

Cihak, R., E. Gutmann, and V. Hanzlikova (1970) Involution and hormoneinduced persistence of the $M$. sphincter (levator) ani in female rats. J. Anat. 106: 93-110.

Dietmer, J. W. (1977) Elcctrical properties of skeletal muscle fibres of the flour moth larva Ephestia kuehniella. J. Insect Physiol. 23: 33-38.

Dominick, O. S., and J. W. Truman (1984) The physiology of wandering behaviour in Manduca sexta. I. Temporal organization and the influence of the internal and external environments. J. Exp. Biol. 110: 35-51.

Ephrussi, B., and G. W. Beadle (1936) A technique of transplantation for Drosophila. Am. Nature 70: 218-225.

Falls, W., and S. Gobel (1979) Golgi and EM studies of the formation of dendritic and axonal arbors: The interneurons of the substantia gelatinosa of Rolando in newborn kittens. J. Comp. Neurol. 187: 1-18.

Finlayson, L. H. (1956) Normal and induced degeneration of abdominal muscles during metamorphosis in the lepidoptera. Q. J. Microsc. Sci. 97: 215-233.

Giebultowicz, J. M., and J. W. Truman (1984) Sexual differentiation in the terminal ganglion of the moth Manduca scxta: Role of sex spccific neuronal death. J. Comp. Neurol. 226: 87-95.

Goodman, C. S., M. Bate, and N. C. Spitzer (1981) Embryonic development of identified neurons: Origin and transformation of the $H$ cell. J. Neurosci. 1: 94-102.

Hamburger, V., and R. W. Oppenheim (1982) Naturally occurring neuronal death in vertebrates. Neurosci. Comment. 1: 39-55.

Jones, W. H., and D. B. Thomas (1962) Changes in the dendritic organization of neurons in the cerebral cortex following deafferentation. J. Anat. 96: 375-381.

Kiguchi, K., and L. M. Riddiford (1978) The role of juvenile hormone in pupal development of the tobacco hornworm, Manduca sexta. J. Insect Physiol. 24: 673-680.

Levi-Montalcini, R. (1982) Developmental neurobiology and the natural history of nerve growth factor. Annu. Rev. Neurosci. 5: 311-362.

Levine, R. B., and J. W. Truman (1982) Metamorphosis of the insect nervous system: Changes in the morphology and synaptic interactions of identified cells. Nature 299. 250-252.

Levine, R. B., and J. W. Truman (1985) Dendritic reorganization of abdominal motorneurons during metamorphosis of the moth, Manduca sexta. J. Neurosci., in press.

Lockshin, R. A., and C. M. Williams (1965) Programmed cell death. I. Cytology 
of degeneration in the intersegmental muscles of the pernyi silkmoth. $J$ Insect Physiol. 11: 123-133.

Meltzer, Y. L. (1971) Hormonal and Attractant Pesticide Technology, Noyes Data Corp., Park Ridge, NJ.

Murphey, R. K., B. Mendenhall, J. Palka, and J. S. Edwards (1975) Deafferentation slows the growth of specific dendrites on identified giant interneurons. J. Comp. Ncurol. 159:407 418.

Nijhout, H. F. (1976) The role of ecdysone in pupation of Manduca sexta. J. Insect Physiol. 22: 453-463.

Nijhout, H. F, and C. M. Williams (1974) Control of moulting and metamorphosis in the tobacco hornworm, Manduca sexta (L.): Cessation of juvenile hormone secretion as a trigger for pupation. J. Exp. Biol. 61: 493-501.

Nuesch, H., and R. F. Stocker (1975) Ultrastructural studies on neuromuscular contacts and the formation of junctions in the flight muscle of Antheraea polyphemus (l ep.). II. Changes after motor nerve section. Cell Tissue Res. 164: $331-355$

Oppenheim, R. W., I. W. Chu-Wang, and J. L. Maderdrut (1978) Cell death of motoneurons in the chick embryo spinal cord. III. The differentiation of motoneurons prior to their induced degeneration following limb-bud re moval. J. Comp. Neurol. 177: 87-112.

Randall, W. C. (1968) Anatomical changes in the neuromuscular complex of the proleg of Galleria mellonella (L.) (Lepidoptera: pyralididae) during metamorphosis. J. Morphol. 125: 105-128.

Randall, W. C. (1970) Ultrastructural changes in the proleg retractor muscles of Galleria mellonella after denervation. J. Insect Physiol. 16: 1927-1943.

Randall, W. C., and R. L. Pipa (1969) Ultrastructural and functional changes during metamorphosis of a proleg muscle and its innervation in Galleria mellonella (L.) (Lepidoptera). J. Morphol. 128: 171-193.

Riddiford, L. M. (1980) Interaction of ecdysteroids and juvenile hormone in the regulation of larval growth and metamorphosis of the tobacco hornworm. in Progress in Ecdysone Research, J. A. Hoffmann, ed., pp. 409430, Elsevier/North-Holland Biomedical Press, Amsterdam.

Riddiford, L. M. (1981) Hormonal control of epidermal cell development. Am. Zool. 21: 751-762.

Riddiford, L. M. (1985) Hormone action on the cellular level. In Comprehensive Insect Physiology, Biochemistry, and Pharmacology, G. A. Kerkut and L. I. Gilbert, eds., Pergamon Press, New York, in press

Runion, H. I. and R. L. Pipa (1970) Electrophysiological and endocrinological correlates during the metamorphic degeneration of a muscle fibre in Galleria mellonella (L.). J. Exp. Biol. 53: 9-24

Schwartz, L. M., and J. W. Truman (1983) Hormonal control of rates of metamorphic development in the tobacco hornworm Manduca sexta. Dev. Biol. 99: 103-114

Schwartz, L. M., and J. W. Truman (1984) Hormonal control of muscie atrophy and degeneration in the moth Antheraea polyphemus. J. Exp. Biol. 111: 13-30

Sedlack, B. J., L. Marchione, B. Devorkin, and R. Davino (1983) Correlations between endocrine gland ultrastructure and hormone titers in the fifth larval instar of Manduca sexta. Gen. Comp. Endocrinol. 52: 291-310.

Stocker, R. F., and $H$. Nuesch (1975) Ultrastructural studies on neuromuscular contacts and the formation of junctions in the flight muscles of Antheraea polyphemus (Lep.). I. Normal adult development. Cell Tissue Res. 159 $245-266$

Sumner, B. E. H., and W. E. Watson (1971) Retraction and cxpansion of the dendritic tree of motor neurones of adult rats induced in vivo. Nature 233 . 273-275

Taylor, II. M. and J. W. Truman (1974) Metamorphosis of the abdominal ganglia of the tobacco hornworm, Manduca sexta. J. Comp. Physiol. 90: $367-388$

Truman, J. W. (1983) Programmed cell death in the nervous system of an adult insect. J. Comp. Neurol. 216: 445-452.

Truman, J. W. (1984) Cell death in invertebrate nervous systems. Annu. Rev. Neuroscl. $7: 1 / 1-188$

Truman, J. W., and L. M. Schwartz (1984) Steroid regulation of neuronal death in the moth nervous system. J. Neurosci. 4: 274-280.

Truman, J. W., D. B. Rountree, S. E. Reiss, and L. M. Schwartz (1983) Ecdysteroids regulate the release and action of eclosion hormone in the tobacco hornworm, Manduca sexta. J. Insect Physiol. 29: 895-900.

Wallace, B. G. (1984) Selective loss of neurites during differentiation of cells in the leech central nervous system. J. Comp. Neurol. 228: 149-153.

Weeks, J. C., and J. W. Truman (1984a) Nel.ural organization of peptideactivated ecdysis behaviors during the metamorphosis of Manduca sexta. I. Conservation of the peristaisis motor pattern at the larval-pupal transformation. J. Comp. Physiol. 155: 407-422.

Weeks, J. C, and J. W. Truman (1984b) Neural organization of peptideactivated ecdysis behaviors during the metamorphosis of Manduca sexta. II. Retention of the proleg motor pattern despite loss of the prolegs at pupation. J. Comp. Physiol. 155: 423-433.

Whitington, P. M., M. Bate, E. Seifert, K. Ridge, and C. S. Goodman (1982) Survival and differentiation of identified embryonic neurons in the absence of their target muscles. Science 215: 973-975.

Zachary, D., and J. A. Hoffmann (1980) Endocrine control of the metamorphosis of the larval muscles in Calliphora erythrocephala (Diptera): In vitro studies of the role of ecdysteroids. Dev. Biol. 80: 235-247. 